\title{
Probing Dry-Weight Improves Left Ventricular Mass Index
}

\author{
Rajiv Agarwal $^{\mathrm{a}, \mathrm{b}}$ J. Michael Bouldin ${ }^{\mathrm{a}}$ Robert P. Light ${ }^{\mathrm{a}}$ Ashok Garg ${ }^{\mathrm{c}}$ \\ a Division of Nephrology, and ${ }^{\mathrm{b}}$ Richard L. Roudebush VA Medical Center, Indianapolis, Ind., and cArizona Heart and \\ Arrythmia Clinic, Phoenix, Ariz., USA
}

\section{Key Words}

Hemodialysis · Hypertension • Ultrafiltration - Ambulatory blood pressure $\cdot$ Volume overload $\cdot$ Echocardiogram $\cdot$ Left ventricular hypertrophy $\cdot$ Left ventricular systolic function

\begin{abstract}
Background: Although probing dry-weight improves blood pressure control, its effect on echocardiographic left ventricular mass index (LVMI) is unknown. Methods: Shortly following dialysis, 292 echocardiograms in 150 patients participating in the DRIP trial were obtained at baseline and longitudinally every 4 weeks on 2 occasions. Results: At baseline, LVMI was $136.3 \mathrm{~g} / \mathrm{m}^{2}$ in the control group and $138.7 \mathrm{~g} / \mathrm{m}^{2}$ in the ultrafiltration group ( $p>0.2$ for difference). The change from baseline in LVMI in the control group was $+3.5 \mathrm{~g} / \mathrm{m}^{2}$ at 4 weeks and $+0.3 \mathrm{~g} / \mathrm{m}^{2}$ at 8 weeks ( $p>0.2$ for both changes). The change from baseline in LVMI in the ultrafiltration group was $-7.4 \mathrm{~g} / \mathrm{m}^{2}$ at 4 weeks $(p=0.005)$ and $-6.3 \mathrm{~g} / \mathrm{m}^{2}$ at 8 weeks $(p=0.045)$. With ultrafiltration, the change in LVMI diameter was $-10.9 \mathrm{~g} / \mathrm{m}^{2}$ more compared to the control group at 4 weeks $(p=0.012)$ and $-6.6 \mathrm{~g} / \mathrm{m}^{2}$ more compared to the control group at 8 weeks $(p=0.21)$. The reduction in interdialytic ambulatory blood pressure was also greater in response to probing dry-weight in those in the top half of LVMI at baseline ( $p=0.02$ for interaction effect at week 8$)$. Conclusion: LVMI, an important determinant of prognosis among long-term dialysis patients, is responsive to probing dry-weight.

Copyright $\odot 2011$ S. Karger AG, Basel
\end{abstract}

\section{Introduction}

It is now well established that left ventricular mass index (LVMI) is a powerful predictor of cardiovascular morbidity and mortality both in the general population $[1,2]$ and in those with hypertension [3]. More recently, the prognostic value of LVMI has been established in patients with chronic kidney disease, including those on long-term dialysis [4-8]. Similarly, left ventricular systolic function has great value for predicting cardiovascular prognosis $[9,10]$. The latter can be assessed by a wellestablished and more sensitive echocardiographic technique that detects systolic dysfunction known as midwall fractional shortening [11]. Midwall fractional shortening is a more objective and sensitive measure of left ventricular systolic function than the semiquantitative assessment of ejection fraction [11, 12]. Midwall fractional shortening has been found to be of prognostic value among dialysis patients [9].

Although both left ventricular mass and function have prognostic significance, it is unclear whether these measures are modifiable among long-term hemodialysis patients. Dietary and dialysate sodium restriction $[13,14]$, more frequent dialysis $[15,16]$, antihypertensive medications $[17,18]$, vitamin $D[19,20]$ and erythropoietin-stimulating agents $[9,21,22]$ have all been used as therapeutic modalities to improve left ventricular mass and function, but probing dry-weight has received little attention [13, 23].

\section{KARGER}

(C) 2011 S. Karger AG, Basel

Fax +41613061234 E-Mail karger@karger.ch www.karger.com www.karger.com/ajn
Prof. Rajiv Agarwal MD

VAMC, $111 \mathrm{~N}$

1481 West 10th St.

Indianapolis, IN 46202 (USA)

Tel. +1 317988 2241, E-Mail ragarwal@iupui.edu 
We have previously demonstrated that probing dryweight can improve interdialytic hypertension within 4 weeks [24]. The reduction in blood pressure persists for at least 8 weeks. However, it is unclear whether left ventricular mass and function is similarly responsive to probing dry-weight. No study has deliberately probed dry-weight in a randomized trial to assess responsiveness of left ventricular mass and function to clinical changes in dry-weight.

Accordingly, the purpose of this study was to evaluate the effect of probing dry-weight on echocardiographic markers of left ventricular mass and function. A further aim was to determine whether these echocardiographic markers are associated with response to interdialytic blood pressure on probing dry-weight.

\section{Methods}

This is a prespecified substudy of the Dry-Weight Reduction in Hypertensive Hemodialysis Patients (DRIP) trial. A detailed protocol and the methods of the study have previously been published [24]. Briefly, we recruited patients 18 years of age or older on long-term hemodialysis for at least 3 months and who were hypertensive based on a mean interdialytic ambulatory blood pressure of 135/85 mm Hg or more. Patients found to have wellcontrolled hypertension had antihypertensive medications withdrawn until they became hypertensive. Patients with stroke, myocardial infarction or limb ischemia in the previous 6 months, ambulatory blood pressure of $>170 / 100 \mathrm{~mm} \mathrm{Hg}$, who had missed more than one dialysis in the prior month, had chronic atrial fibrillation or morbid obesity (BMI $>40)$ were excluded.

After a six-hemodialysis run-in phase, at which time baseline data were collected, patients were randomized in a 1:2 proportion into a control group and ultrafiltration trial group for 8 weeks. During this 24-dialysis treatment phase, patients were seen at each dialysis visit and had evaluation of dry-weight and symptoms and signs related to hypovolemia by study personnel.

Randomization to treatment or control groups was carried out in permuted blocks with a 2:1 ultrafiltration:control ratio. Opaque sealed envelopes were used for treatment allocation by study personnel after assuring that the inclusion-exclusion criteria were met.

The study protocol was approved by the institutional review boards and the VA Research and Development Committee; all patients provided written informed consent. The trial was registered at ClinicalTrials.gov (NCT00067665).

\section{Ambulatory Blood Pressure Monitoring}

Ambulatory blood pressure monitoring was performed after the midweek hemodialysis session for $44 \mathrm{~h}$. Blood pressures were recorded every 20 min during the day (6 a.m. to 10 p.m.) and every 30 min during the night (10 p.m. to 6 a.m.) using a Spacelab 90207 ABP monitor (SpaceLabs Medical Inc., Redmond, Wash., USA) in the nonaccess arm. Recordings began immediately after hemodialysis and terminated immediately before the subsequent dialysis.
The accuracy of ambulatory blood pressure recordings was confirmed against auscultated blood pressure at baseline. Hourly means were calculated. These means were then averaged over the entire course of the recording to provide systolic and diastolic interdialytic ambulatory blood pressures.

\section{Echocardiograms}

Two-dimensional guided M-mode echocardiograms were performed by dedicated technicians 30-60 min following dialysis in the dialysis unit with a digital cardiac ultrasound machine (Cypress Acuson, Siemens Medical). The postdialysis period was selected for echocardiography as it allows control over volume state of the patient since it is associated with the least intravascular volume.

The protocol specified the recording of at least six cycles of 2-dimensional parasternal long- and short-axis left ventricular views with optimal orientation of the cursor beam to derive additional M-mode recordings. Each patient underwent six Mmode measurements using standards of the American Society of Echocardiography [25] and LVMI and midwall fractional shortening was measured as previously described $[10,26]$. All measurements were made over six cardiac cycles by a highly skilled echocardiographer and confirmed by an experienced cardiologist.

\section{Statistical Analysis}

Data were first analyzed by graphical methods. A mixed model accounting for repeated measurements was fitted for several outcome echocardiographic parameters of interest, such as LVMI, left ventricular internal diameter, left ventricular posterior wall thickness, intraventricular septal thickness and midwall fractional shortening. The effect of intervention (ultrafiltration vs. control), time (baseline, 4 weeks and 8 weeks) and their interaction was tested and $95 \%$ confidence intervals calculated using maximal likelihood estimates. The random part of the equation used subject and visits modeled using an unstructured covariance matrix. Since four different echocardiographers performed these measurements, a random effect was used for the echocardiographer.

To analyze the effect of baseline echocardiographic parameters on interdialytic blood pressure, we first dichotomized the baseline echocardiographic parameter (e.g. LVMI) at the median. The median of the parameter was calculated using all the echocardiograms performed at the baseline visit. We then carried this assignment forward to the week 4 and week 8 visits. A similar mixed-effects model as reported above was used. The fixed part of the model had interdialytic systolic blood pressure as an outcome variable. The predictors were the echocardiographic variable (indicator variable dichotomized about the median), intervention, time and all possible interactions of these three indicator variables. The three-way interaction indicated whether the echocardiographic variable predicted the blood pressure response.

To analyze the effect of time-varying echocardiographic parameters on interdialytic blood pressure, we first dichotomized the baseline echocardiographic parameter (e.g. LVMI) at the median. The median of the parameter was calculated using all the echocardiograms performed at the baseline visit. We then assigned all echocardiograms to a dichotomous category at the week 4 and week 8 visits. A similar mixed-effects model as reported above was used. The fixed part of the model had interdialytic systolic blood pressure as an outcome variable. The predictors were 
Table 1. Subject characteristics

\begin{tabular}{|c|c|c|c|c|}
\hline Clinical characteristic & Control & Ultrafiltration & Total & $\mathrm{p}$ \\
\hline Number & $39(35)$ & $74(65)$ & $113(100)$ & \\
\hline Age, years & $55.5 \pm 11.5$ & $54.3 \pm 12.6$ & $54.7 \pm 12.2$ & 0.6 \\
\hline Male & $31(79)$ & $48(65)$ & $79(70)$ & 0.1 \\
\hline Race & & & & 0.8 \\
\hline White & $3(8)$ & $9(12)$ & $12(11)$ & \\
\hline Black & $35(90)$ & $63(85)$ & $98(87)$ & \\
\hline Other & $1(3)$ & $2(3)$ & $3(3)$ & \\
\hline Pre-HD seated BP, mm Hg & $157.8 \pm 15.8 / 87.0 \pm 12.8$ & $158.5 \pm 16.2 / 85.5 \pm 10.5$ & $158.3 \pm 16.0 / 86.0 \pm 11.3$ & $0.8 / 0.5$ \\
\hline Post-HD seated SBP, mm Hg & $141.5 \pm 19.9 / 77.1 \pm 13.2$ & $142.9 \pm 17.6 / 78.0 \pm 10.1$ & $142.4 \pm 18.3 / 77.7 \pm 11.2$ & $0.7 / 0.7$ \\
\hline Pre-HD weight, kg & $82.8 \pm 16.7$ & $82.2 \pm 20.0$ & $82.4 \pm 18.8$ & 0.9 \\
\hline Post-HD weight, $\mathrm{kg}$ & $80.0 \pm 16.1$ & $79.2 \pm 19.2$ & $79.5 \pm 18.1$ & 0.8 \\
\hline BMI & $26.7 \pm 5.9$ & $26.8 \pm 5.7$ & $26.7 \pm 5.7$ & 0.9 \\
\hline Years on dialysis & $4.3 \pm 6.0$ & $3.6 \pm 4.2$ & $3.9 \pm 4.9$ & 0.5 \\
\hline Etiology of ESRD & & & & 0.7 \\
\hline Diabetes mellitus & $15(38)$ & $31(42)$ & $46(41)$ & \\
\hline Hypertension & $18(46)$ & $31(42)$ & $49(43)$ & \\
\hline Glomerulonephritis & $2(5)$ & $3(4)$ & $5(4)$ & \\
\hline Polycystic kidney disease & 0 & $3(4)$ & $3(3)$ & \\
\hline Other & $4(10)$ & $6(8)$ & $10(9)$ & \\
\hline Current smoker & $15(38)$ & $23(31)$ & $38(34)$ & 0.4 \\
\hline \multicolumn{5}{|l|}{ History of } \\
\hline Congestive heart failure & $4(10)$ & $15(20)$ & $19(17)$ & 0.2 \\
\hline Myocardial infarction & $6(15)$ & $13(18)$ & $19(17)$ & 0.8 \\
\hline Stroke & $4(10)$ & $7(9)$ & $11(10)$ & 0.9 \\
\hline Urea reduction ratio, $\%$ & $73.0 \pm 6.3$ & $74.2 \pm 7.4$ & $73.8 \pm 7.0$ & 0.4 \\
\hline Albumin, g/dl & $3.8 \pm 0.4$ & $3.7 \pm 0.5$ & $3.7 \pm 0.5$ & 0.9 \\
\hline Hemoglobin, g/dl & $12.1 \pm 1.4$ & $12.2 \pm 1.1$ & $12.2 \pm 1.2$ & 0.7 \\
\hline Presence of pedal edema & $7(18)$ & $16(22)$ & $23(20)$ & 0.6 \\
\hline Number receiving antihypertensive drugs & $29(74)$ & $63(85)$ & $92(81)$ & 0.2 \\
\hline Number of antihypertensives in users & $2.1 \pm 1.7$ & $2.2 \pm 1.6$ & $2.1 \pm 1.6$ & 0.8 \\
\hline Dihydropyridine calcium channel blockers & $16(41)$ & $33(45)$ & $49(43)$ & 0.7 \\
\hline Nondihydropyridine calcium channel blockers & $2(5)$ & $4(5)$ & $6(5)$ & 0.9 \\
\hline$\beta$-Blockers & $25(64)$ & $50(68)$ & $75(66)$ & 0.7 \\
\hline$\alpha$-Blockers & $3(8)$ & $5(7)$ & $8(7)$ & 0.9 \\
\hline Centrally acting agents & $8(21)$ & $21(28)$ & $29(26)$ & 0.4 \\
\hline Vasodilators & $9(23)$ & $10(14)$ & $19(17)$ & 0.2 \\
\hline ACE inhibitors & $20(51)$ & $38(51)$ & $58(51)$ & 1 \\
\hline Angiotensin receptor blockers & $4(10)$ & $14(19)$ & $18(16)$ & 0.2 \\
\hline
\end{tabular}

Figures in parentheses represent percentages. $\mathrm{HD}=$ Hemodialysis; $\mathrm{SBP}=$ systolic blood pressure; ESRD = end-stage renal disease.

the echocardiographic variable (indicator variable dichotomized about the median), intervention, time and all possible interactions of these three indicator variables. We next calculated the transitional change from a low and high category of the echocardiographic variable in the ultrafiltration group and control groups. We then calculated the differences between these changes. Finally, we tested the significance of the differences using the Wald test.

The nominal level of significance was set at a two-sided $p$ value of $<0.05$ and all statistical analyses were performed with Stata version 11 (StataCorp LP, College Station, Tex., USA).

\section{Results}

Between March 2004 and April 2008, we randomized 100 patients to the ultrafiltration group and 50 patients to the control group. In the ultrafiltration group, 192 echocardiograms were performed on the 100 patients (72 at baseline), and 100 echocardiograms were performed on the 50 patients of the control group (38 at baseline). The trial flow of these participants has previously been 
Table 2. Echocardiographic structure and function and their change within and between groups over time

\begin{tabular}{|c|c|c|c|c|c|c|c|c|c|c|c|c|c|}
\hline \multirow{2}{*}{$\begin{array}{l}\text { Echocardio- } \\
\text { graphic volume } \\
\text { parameter }\end{array}$} & \multirow{2}{*}{$\begin{array}{l}\text { Intervention } \\
\text { group }\end{array}$} & \multicolumn{4}{|c|}{ Baseline } & \multicolumn{4}{|c|}{ Week 4 change from baseline } & \multicolumn{4}{|c|}{ Week 8 change from baseline } \\
\hline & & $\mathrm{n}$ & mean & $95 \% \mathrm{CI}$ & $\mathrm{p}$ & $\mathrm{n}$ & mean & $95 \% \mathrm{CI}$ & $\mathrm{p}$ & $\mathrm{n}$ & mean & $95 \% \mathrm{CI}$ & $\mathrm{p}$ \\
\hline \multirow[t]{3}{*}{ LVMI, $\mathrm{g} / \mathrm{m}^{2}$} & control & 38 & 136.3 & $125.1,147.5$ & & 31 & 3.5 & $-3.3,10.3$ & $>0.2$ & 31 & 0.3 & $-8.1,8.8$ & $>0.2$ \\
\hline & UF & 72 & 138.7 & $130.6,146.8$ & & 57 & -7.4 & $-12.5,-2.3$ & $0.005^{*}$ & 63 & -6.3 & $-12.5,-0.1$ & $0.045^{*}$ \\
\hline & UF - control & & 2.5 & $-11.4,16.3$ & $>0.2$ & & -10.9 & $-19.4,-2.4$ & $0.012^{*}$ & & -6.6 & $-17.1,3.8$ & $>0.2$ \\
\hline \multirow[t]{3}{*}{ IVSd, $\mathrm{cm}$} & control & 38 & 1.31 & $1.24,1.37$ & & 31 & 0.02 & $-0.01,0.05$ & $>0.2$ & 31 & 0 & $-0.04,0.03$ & $>0.2$ \\
\hline & UF & 72 & 1.32 & $1.27,1.37$ & & 57 & 0.01 & $-0.01,0.03$ & $>0.2$ & 63 & 0.01 & $-0.02,0.04$ & $>0.2$ \\
\hline & UF - control & & 0.01 & $-0.06,0.09$ & $>0.2$ & & -0.01 & $-0.05,0.02$ & $>0.2$ & & 0.01 & $-0.03,0.06$ & $>0.2$ \\
\hline \multirow[t]{3}{*}{ LVId, cm } & control & 38 & 5.05 & $4.84,5.26$ & & 31 & -0.01 & $-0.16,0.14$ & $>0.2$ & 31 & -0.06 & $-0.22,0.11$ & $>0.2$ \\
\hline & UF & 72 & 4.98 & $4.83,5.13$ & & 57 & -0.22 & $-0.33,-0.11$ & $<0.001^{*}$ & 63 & -0.19 & $-0.31,-0.07$ & $0.002^{*}$ \\
\hline & UF - control & & -0.07 & $-0.33,0.19$ & $>0.2$ & & -0.21 & $-0.40,-0.02$ & $0.030^{*}$ & & -0.13 & $-0.34,0.07$ & $>0.2$ \\
\hline \multirow[t]{3}{*}{ LVPWd, cm } & control & 38 & 1.25 & $1.20,1.31$ & & 31 & 0.04 & $0.00,0.07$ & $0.025^{*}$ & 31 & 0.04 & $0.01,0.07$ & $0.016^{*}$ \\
\hline & UF & 72 & 1.29 & $1.25,1.33$ & & 57 & 0.01 & $-0.01,0.03$ & $>0.2$ & 63 & 0.01 & $-0.01,0.03$ & $>0.2$ \\
\hline & UF - control & & 0.04 & $-0.03,0.10$ & $>0.2$ & & -0.03 & $-0.07,0.01$ & 0.19 & & -0.03 & $-0.07,0.01$ & 0.13 \\
\hline \multirow[t]{3}{*}{ MWFS, \% } & control & 38 & 13.93 & $12.33,15.54$ & & 31 & 0.39 & $-0.22,0.99$ & $>0.2$ & 31 & -0.01 & $-0.68,0.65$ & $>0.2$ \\
\hline & UF & 72 & 13.42 & $11.92,14.91$ & & 57 & -0.24 & $-0.69,0.20$ & $>0.2$ & 63 & -0.31 & $-0.79,0.17$ & $>0.2$ \\
\hline & UF - control & & -0.52 & $-1.50,0.46$ & $>0.2$ & & -0.63 & $-1.39,0.12$ & 0.09 & & -0.3 & $-1.12,0.52$ & $>0.2$ \\
\hline
\end{tabular}

IVSd = Intraventricular septal thickness at diastole; LVId = left ventricular internal diameter at diastole; LVPWd = left ventricular posterior wall thickness at diastole; MWFS $=$ midwall fractional shortening; $\mathrm{UF}=$ ultrafiltration. ${ }^{*} \mathrm{p}<0.05$.

described [24]. The two treatment groups of patients who had echocardiograms were well balanced with respect to the baseline characteristics (table 1).

Table 2 shows the echocardiographic characteristics at baseline and the changes at 4 weeks and 8 weeks in the control and ultrafiltration groups. Baseline LVMI was $136.3 \mathrm{~g} / \mathrm{m}^{2}$ in the control group and $138.7 \mathrm{~g} / \mathrm{m}^{2}$ in the ultrafiltration group. In the control group, LVMI increased by $3.5 \mathrm{~g} / \mathrm{m}^{2}$ at 4 weeks and $0.3 \mathrm{~g} / \mathrm{m}^{2}$ at 8 weeks. In the ultrafiltration group, LVMI decreased by $7.4 \mathrm{~g} / \mathrm{m}^{2}$ at 4 weeks and $6.3 \mathrm{~g} / \mathrm{m}^{2}$ at 8 weeks. As a result, with ultrafiltration, change in LVMI was $-10.9 \mathrm{~g} / \mathrm{m}^{2}(\mathrm{p}=0.012)$ more compared to the control group at 4 weeks, and $-6.6 \mathrm{~g} / \mathrm{m}^{2}$ more compared to the control group at 8 weeks $(\mathrm{p}=0.21)$.

From baseline, the left ventricular interior diameter of $4.98 \mathrm{~cm}$ ultrafiltration-induced change was $-0.21 \mathrm{~cm}$ more at 4 weeks $(\mathrm{p}=0.03)$ and $-0.19 \mathrm{~cm}$ more at 8 weeks $(p=0.21)$. The changes in left ventricular posterior wall thickness, intraventricular septal thickness and midwall fractional shortening were not significant between groups over time.

Figure 1 shows the reduction in interdialytic ambulatory blood pressure as a function of five echocardiographic volume parameters dichotomized at the median value at the baseline visit. For example, in the case of
LVMI, at 8 weeks the mean reduction in systolic ambulatory blood pressure in the ultrafiltration group was 2.5 $\mathrm{mm} \mathrm{Hg}$ greater compared to the control group in those with a baseline LVMI below the median. In contrast, the mean reduction in systolic ambulatory blood pressure in the ultrafitration group was $15.7 \mathrm{~mm} \mathrm{Hg}$ greater in those with baseline LVMI above the median (and therefore presumably more volume overload). The difference between $2.5 \mathrm{~mm} \mathrm{Hg}$ increase and $15.7 \mathrm{~mm} \mathrm{Hg}$ reduction was statistically significant $(p=0.02)$. Although not statistically significant, at 4 weeks, the results showed greater reduction $(5.6 \mathrm{~mm} \mathrm{Hg}$ ) in the high-LVMI group (which presumably is more volume-overloaded at baseline) compared to the low-index group $(0.3 \mathrm{~mm} \mathrm{Hg})$. Neither the components of LVMI nor midwall fractional shortening (a sensitive index of left ventricular systolic function) were related to blood pressure response.

Figure 2 shows the reduction in interdialytic ambulatory blood pressure as a function of three echocardiographic volume parameters dichotomized at the median value at the baseline visit. This dichotomized value was then used to grade change in indices at 4 and 8 weeks. Only the 8-week results are shown since the 4-week results looked even less significant. As in the baseline model shown in figure 1, the results show that none of the 


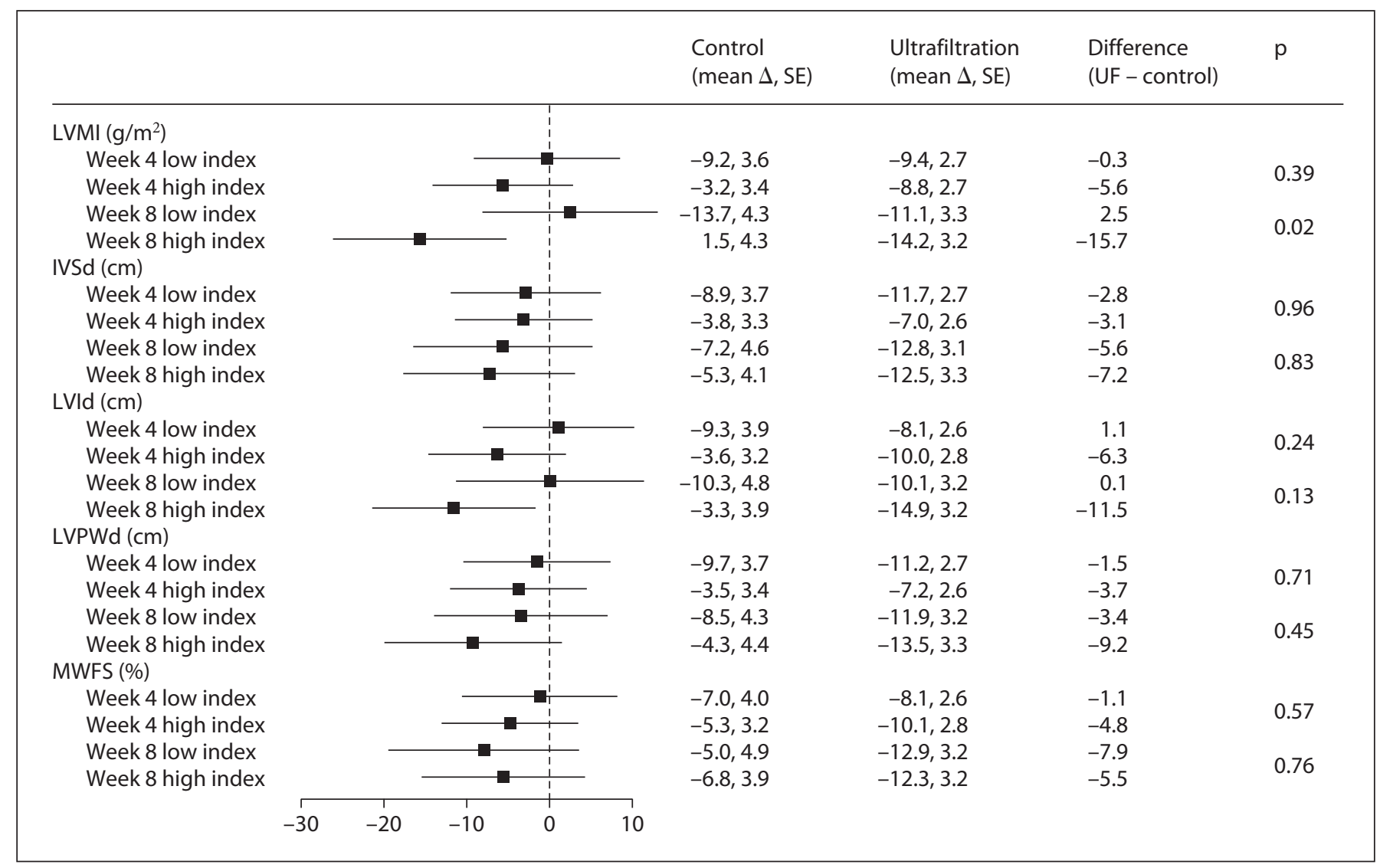

Fig. 1. Changes in 44-hour interdialytic systolic blood pressure as a function of the echocardiographic volume parameter. The echocardiographic volume parameter was dichotomized at the median value at the baseline visit, yielding a low index and a high index. Compared to the high index, low index would be expected to have less volume. The forest plot shows the additional change in systolic blood pressure in the ultrafiltration group compared to the change in the control group. The mean additional change in blood pressure (shown in the forest plot) in the ultrafiltration group at 4 and 8 weeks did not differ between low index and high index. IVSd = Intraventricular septal thickness at diastole; LVId = left ventricular interior diameter at diastole; LVPWd = left ventricular posterior wall thickness at diastole; $\mathrm{MWFS}=$ midwall fractional shortening. echocardiographic parameters predicted the ultrafiltration-induced change in systolic blood pressure at 8 weeks. Neither at 4 weeks (data not shown) nor at 8 weeks (fig. 2) were the interaction values between the high and low indices significant.

\section{Discussion}

LVMI is a potent indicator of prognosis both in patients with hypertension and those with chronic kidney disease, including those on hemodialysis [1-8]. Midwall fractional shortening is a sensitive index of left ventricular systolic function and is also a powerful prognostic marker among hemodialysis patients $[9,11,12]$. Our study demonstrates that regression of left ventricular mass can be effected by probing dry-weight. However, midwall fractional shortening was not affected. Furthermore, it appears that the reduction in interdialytic systolic blood pressure may be more in those with a higher LVMI. This indicates that, in part, LVMI is indicative of volume overload as reflected by left ventricular chamber dilatation. To support this notion, we found that probing dry-weight reduced left ventricular chamber dilatation, but did not affect left ventricular wall thickness. To the extent this notion is true, increased LVMI among dialysis patients may reflect excess volume. However, the change in echocardiographic LVMI or its components or change in midwall fractional shortening does not appear to predict the blood pressure response to probing dry-weight. 


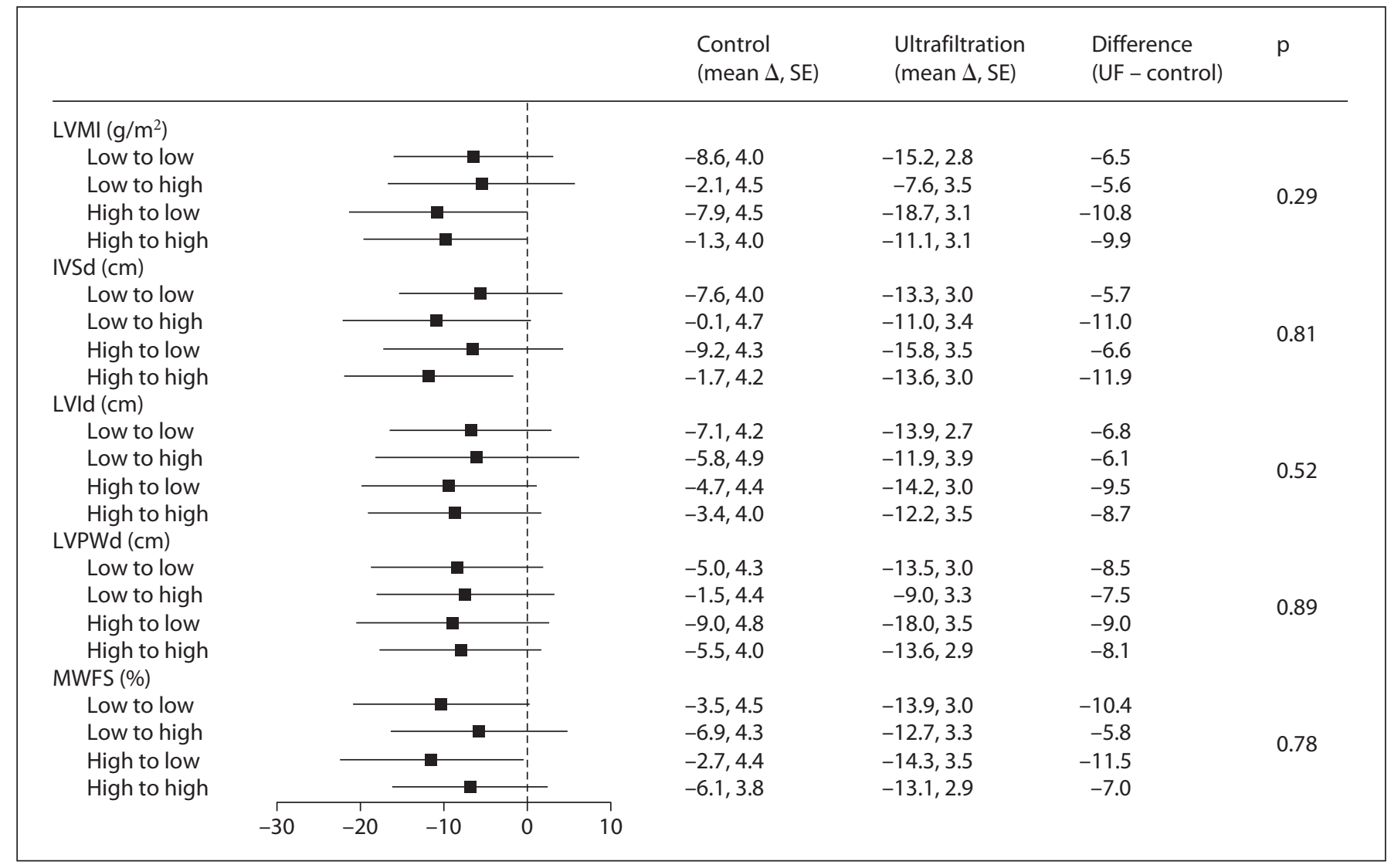

Fig. 2. Changes in 44-hour interdialytic systolic blood pressure as a function of change in the echocardiographic volume parameter. As in figure 1, the echocardiographic volume parameter was dichotomized at the median value at the baseline visit, yielding a low index and a high index. This value was then used to classify patients into low- or high-index groups at 4 weeks and 8 weeks. The forest plot shows the 8 -week additional change from baseline in systolic blood pressure in the ultrafiltration group compared to the control group. Blood pressure change evoked by the transition in volume from low to high was similar to that from low to low. Similarly, blood pressure change evoked by the transition in volume from high to low was similar to that from high to high. Likewise, transitions in volume from low to either volume were not different from transitions from high to either volume. Thus, changes in volume state were not predictive of change in blood pressure. Abbreviations are defined as in figure 1.
LVMI reduced rapidly - more rapidly than what would be expected in the general population. The reduction in LVMI was most likely due to reduction in left ventricular internal diameter. Due to the geometric assumptions underlying the LVMI calculations, reduction in left ventricular diameter will most likely lead to improvement in LVMI. It is very unlikely that reduction in blood pressure by itself (in the absence of volume correction) would be associated with improvement in LVMI.

It may appear internally inconsistent that a higher LVMI was associated with a greater reduction in interdialytic systolic blood pressure, yet reduction in LVMI was not associated with changes in interdialytic systolic blood pressure. The latter negative finding is likely be- cause our study was not powered adequately to discover a relationship between change in LVMI with probing dryweight and change in interdialytic systolic blood pressure. On the other hand, it is possible that the association between higher LVMI and greater reduction in interdialytic ambulatory systolic blood pressure may simply be a chance finding. However, given that the relationship between higher LVMI and a greater reduction in systolic blood pressure is physiologically plausible, it is more likely that the former scenario of an underpowered study is more likely.

LVMI, as assessed in this study in the postdialysis state, can be routinely evaluated using standard echocardiographic techniques. Our study found that patients 
who had a higher LVMI achieved a greater reduction in interdialytic ambulatory systolic blood pressure. Thus, patients on long-term dialysis with hypertension, especially those with a higher LVMI, should have their dryweight probed as an initial intervention as a way to improve prognosis. On the other hand, midwall fractional shortening, an objective measure of left ventricular systolic function, had no relationship with blood pressure lowering upon probing dry-weight. This finding should not be taken to imply that those with very poor systolic function, who were largely excluded from our study, cannot experience an improvement upon probing dryweight.

The recently completed and published daily dialysis study of the Frequent Hemodialysis Network demonstrated that daily dialysis was associated with an approximately 10-gram improvement in left ventricular mass and about $10-\mathrm{mm} \mathrm{Hg}$ lowering in systolic blood pressure [27]. The results of our study show an improvement within 8 weeks of a similar magnitude in both left ventricular mass and systolic pressure. It is possible that as in our study, frequent dialysis simply has salutary effects through achieving dry-weight over a period of time. To the extent this is true, probing dry-weight may be a simpler expedient than frequent dialysis to improve outcomes related to volume excess such as heart failure and strokes.

One strength of our study was that certified technicians performed the serial echocardiograms in the dialysis unit using a prespecified study protocol in the context of a randomized trial. Our study does, however, have some limitations. Although the analysis of LVMI was prespecified, patients were not randomized based on LVMI. In the absence of randomization based on LVMI, we cannot establish a cause-and-effect relationship between LVMI and subsequent blood pressure reduction. Before performing echocardiograms, we could have waited longer after dialysis for fluid equilibration to occur. However, this would have made our study less feasible. Finally, there were few non-African-American patients in our study. Whether the results of our study are generalizable to non-African-American patients will need to be demonstrated in future studies.

In conclusion, among chronic hemodialysis patients, LVMI, a powerful indicator of mortality, can be reduced by probing dry-weight. If probing dry-weight is causally related to improvement in LVMI, this simple and effective tool has the potential to improve dismal cardiovascular outcomes. Our study provides another reason why nephrologists should remain vigilant in continuously assessing and managing dry-weight among their long-term dialysis patients.

\section{Acknowledgments}

We are indebted to the participating hemodialysis patients who volunteered their time; the staff of the dialysis units at Dialysis Clinics Inc., Clarian Health and the Roudebush VA Medical Center; the faculty of the Division of Nephrology for allowing us to study their patients; the research technicians, research fellows and members of the Data and Safety Monitoring Board. This work was supported by grant No. 5RO1-DK062030-07 from the National Institutes of Health.

\section{Disclosure Statement}

None.

\section{References}

1 Kannel WB: Left ventricular hypertrophy as a risk factor: the Framingham experience. J Hypertens Suppl 1991;9:S3-S8.

$\checkmark 2$ Levy D, Garrison RJ, Savage DD, Kannel WB, Castelli WP: Prognostic implications of echocardiographically determined left ventricular mass in the Framingham Heart Study. N Engl J Med 1990;322:1561-1566.

-3 Devereux RB, Wachtell K, Gerdts E, Boman K, Nieminen MS, Papademetriou V, Rokkedal J, Harris K, Aurup P, Dahlof B: Prognostic significance of left ventricular mass change during treatment of hypertension. JAMA 2004;292:2350-2356.

Dry-Weight Reduction Improves LVMI
-4 Levin A, Singer J, Thompson CR, Ross H, Lewis M: Prevalent left ventricular hypertrophy in the predialysis population: identifying opportunities for intervention. Am J Kidney Dis 1996;27:347-354.

5 Levin A, Thompson CR, Ethier J, Carlisle EJ, Tobe S, Mendelssohn D, Burgess E, Jindal K, Barrett B, Singer J, Djurdjev O: Left ventricular mass index increase in early renal disease: impact of decline in hemoglobin. Am J Kidney Dis 1999;34:125-134.

\footnotetext{
6 Paoletti E, Cassottana P, Bellino D, Specchia C, Messa P, Cannella G: Left ventricular geometry and adverse cardiovascular events in chronic hemodialysis patients on prolonged therapy with ACE inhibitors. Am J Kidney Dis 2002;40:728-736.

$\checkmark 7$ London GM, Pannier B, Guerin AP, Blacher J, Marchais SJ, Darne B, Metivier F, Adda H, Safar ME: Alterations of left ventricular hypertrophy in and survival of patients receiving hemodialysis: follow-up of an interventional study. J Am Soc Nephrol 2001;12: 2759-2767.
} 
-8 Zoccali C, Benedetto FA, Mallamaci F, Tripepi G, Giacone G, Cataliotti A, Seminara G, Stancanelli B, Malatino LS: Prognostic impact of the indexation of left ventricular mass in patients undergoing dialysis. J Am Soc Nephrol 2001;12:2768-2774.

$\checkmark 9$ Levin A, Djurdjev O, Thompson C, Barrett B, Ethier J, Carlisle E, Barre P, Magner P, Muirhead N, Tobe S, Tam P, Wadgymar JA, Kappel J, Holland D, Pichette V, Shoker A, Soltys G, Verrelli M, Singer J: Canadian randomized trial of hemoglobin maintenance to prevent or delay left ventricular mass growth in patients with CKD. Am J Kidney Dis 2005; 46:799-811.

10 Satyan S, Light RP, Agarwal R: Relationships of N-terminal pro-B-natriuretic peptide and cardiac troponin $\mathrm{T}$ to left ventricular mass and function and mortality in asymptomatic hemodialysis patients. Am J Kidney Dis 2007;50:1009-1019.

-11 Aurigemma GP, Silver KH, Priest MA, Gaasch WH: Geometric changes allow normal ejection fraction despite depressed myocardial shortening in hypertensive left ventricular hypertrophy. J Am Coll Cardiol 1995;26:195-202.

-12 Vinch CS, Aurigemma GP, Simon HU, Hill JC, Tighe DA, Meyer TE: Analysis of left ventricular systolic function using midwall mechanics in patients $>60$ years of age with hypertensive heart disease and heart failure. Am J Cardiol 2005;96:1299-1303.

13 Ozkahya M, Ok E, Cirit M, Aydin S, Akcicek F, Basci A, Dorhout Mees EJ: Regression of left ventricular hypertrophy in haemodialysis patients by ultrafiltration and reduced salt intake without antihypertensive drugs. Nephrol Dial Transplant 1998;13:1489-1493.
14 Kayikcioglu M, Tumuklu M, Ozkahya M, Ozdogan O, Asci G, Duman S, Toz H, Can LH, Basci A, Ok E: The benefit of salt restriction in the treatment of end-stage renal disease by haemodialysis. Nephrol Dial Transplant 2009;24:956-962.

15 Ayus JC, Mizani MR, Achinger SG, Thadhani R, Go AS, Lee S: Effects of short daily versus conventional hemodialysis on left ventricular hypertrophy and inflammatory markers: a prospective, controlled study. J Am Soc Nephrol 2005;16:2778-2788.

16 Chan CT, Floras JS, Miller JA, Richardson RM, Pierratos A: Regression of left ventricular hypertrophy after conversion to nocturnal hemodialysis. Kidney Int 2002;61:22352239.

17 Cannella G, Paoletti E, Delfino R, Peloso G, Rolla D, Molinari S: Prolonged therapy with ACE inhibitors induces a regression of left ventricular hypertrophy of dialyzed uremic patients independently from hypotensive effects. Am J Kidney Dis 1997;30:659-664.

18 Cannella G, Paoletti E, Delfino R, Peloso G Molinari S, Traverso GB: Regression of left ventricular hypertrophy in hypertensive dialyzed uremic patients on long-term antihypertensive therapy. Kidney Int 1993;44:881886.

19 Park CW, Oh YS, Shin YS, Kim CM, Kim YS, Kim SY, Choi EJ, Chang YS, Bang BK: Intravenous calcitriol regresses myocardial hypertrophy in hemodialysis patients with secondary hyperparathyroidism. Am J Kidney Dis 1999;33:73-81.

20 Bodyak N, Ayus JC, Achinger S, Shivalingappa V, Ke Q, Chen YS, Rigor DL, Stillman I, Tamez H, Kroeger PE, Wu-Wong RR, Karumanchi SA, Thadhani R, Kang PM: Activated vitamin $D$ attenuates left ventricular abnormalities induced by dietary sodium in Dahl salt-sensitive animals. Proc Natl Acad Sci USA 2007;104:16810-16815.
21 Cannella G, La Canna G, Sandrini M, Gaggiotti M, Nordio G, Movilli E, Mombelloni S, Visioli O, Maiorca R: Reversal of left ventricular hypertrophy following recombinant human erythropoietin treatment of anaemic dialysed uraemic patients. Nephrol Dial Transplant 1991;6:31-37.

-22 Roger SD, McMahon LP, Clarkson A, Disney A, Harris D, Hawley C, Healy H, Kerr P, Lynn K, Parnham A, Pascoe R, Voss D, Walker R, Levin A: Effects of early and late intervention with epoetin alpha on left ventricular mass among patients with chronic kidney disease (stage 3 or 4): results of a randomized clinical trial. J Am Soc Nephrol 2004;15:148-156.

23 Ozkahya M, Toz H, Qzerkan F, Duman S, Ok E, Basci A, Mees EJ: Impact of volume control on left ventricular hypertrophy in dialysis patients. J Nephrol 2002;15:655-660.

24 Agarwal R, Alborzi P, Satyan S, Light RP: Dry-weight reduction in hypertensive hemodialysis patients (DRIP): a randomized, controlled trial. Hypertension 2009;53:500-507.

25 Sahn DJ, DeMaria A, Kisslo J, Weyman A: Recommendations regarding quantitation in M-mode echocardiography: results of a survey of echocardiographic measurements. Circulation 1978;58:1072-1083.

26 Agarwal R, Brim NJ, Mahenthiran J, Andersen MJ, Saha C: Out-of-hemodialysis-unit blood pressure is a superior determinant of left ventricular hypertrophy. Hypertension 2006;47:62-68.

$>27$ Chertow GM, Levin NW, Beck GJ, Depner TA, Eggers PW, Gassman JJ, Gorodetskaya I, Greene T, James S, Larive B, Lindsay RM, Mehta RL, Miller B, Ornt DB, Rajagopalan S, Rastogi A, Rocco MV, Schiller B, Sergeyeva O, Schulman G, Ting GO, Unruh ML, Star RA, Kliger AS: In-center hemodialysis six times per week versus three times per week. N Engl J Med 2010;363:2287-2300. 